\title{
RESONANCE BETWEEN CANTOR SETS
}

\author{
YUVAL PERES AND PABLO SHMERKIN
}

\begin{abstract}
Let $C_{a}$ be the central Cantor set obtained by removing a central interval of length $1-2 a$ from the unit interval, and continuing this process inductively on each of the remaining two intervals. We prove that if $\log b / \log a$ is irrational, then

$$
\operatorname{dim}\left(C_{a}+C_{b}\right)=\min \left(\operatorname{dim}\left(C_{a}\right)+\operatorname{dim}\left(C_{b}\right), 1\right),
$$

where dim is Hausdorff dimension. More generally, given two self-similar sets $K, K^{\prime}$ in $\mathbb{R}$ and a scaling parameter $s>0$, if the dimension of the arithmetic sum $K+s K^{\prime}$ is strictly smaller than $\operatorname{dim}(K)+\operatorname{dim}\left(K^{\prime}\right) \leq 1$ ("geometric resonance"), then there exists $r<1$ such that all contraction ratios of the similitudes defining $K$ and $K^{\prime}$ are powers of $r$ ("algebraic resonance"). Our method also yields a new result on the projections of planar self-similar sets generated by an iterated function system that includes a scaled irrational rotation.
\end{abstract}

\section{IntRoduCtion AND STATEMENT OF RESUlts}

In many situations in dynamical systems and geometric measure theory one encounters results which are valid for almost every member of a parametrized family. In general, those results are sharp in the sense that the set of exceptions may have positive, or even full, dimension. But when the constructions are done in a dynamical or geometrically regular way, there is often some countable set of parameters, usually arising from an algebraic condition, where the result fails, and it is natural to conjecture that these are all parameters for which the result fails, see for example [6], [8], 20], [18], [7. However, there are very few cases where the set of exceptions has been explicitly determined. In this paper we will find the exact set of parameters for which certain kind of resonance between two self-similar sets occurs. This set is countable and given by a simple, natural algebraic relation.

Let $C_{a}$ be the central Cantor set obtained by removing a middle interval of length $1-2 a$ from the unit interval, and continuing this process inductively on each of the remaining intervals. Explicitly,

$$
C_{a}=\left\{(1-a) \sum_{i=0}^{\infty} \omega_{i} a^{i}: \omega_{i} \in\{0,1\}\right\} .
$$

It is well known that $\operatorname{dim}\left(C_{a}\right)=\log 2 / \log (1 / a)$.

Research of Y. Peres supported in part by NSF grant DMS-0605166.

Research of P. Shmerkin supported in part by the Academy of Finland and Microsoft Research. 
Our main result is the following:

Theorem 1. If $\log b / \log a$ is irrational, then

$$
\operatorname{dim}\left(C_{a}+C_{b}\right)=\min \left(\operatorname{dim}\left(C_{a}\right)+\operatorname{dim}\left(C_{b}\right), 1\right) .
$$

When $\log b / \log a$ is rational and $\operatorname{dim}\left(C_{a}\right)+\operatorname{dim}\left(C_{b}\right) \leq 1$, the equality (11) does not hold. This is well known; we sketch a proof after (4) below.

An iterated function system (or i.f.s. for short) on a complete metric space $\mathbb{X}$ is a finite collection of maps $\left\{f_{1}, \ldots, f_{n}\right\}$ from $\mathbb{X}$ to itself, such that all $f_{j}$ are contractions (have Lipschitz constant strictly less than 1.) The attractor of this i.f.s. is the unique nonempty compact set $E$ such that

$$
E=\bigcup_{i=1}^{n} f_{i}(E) .
$$

In particular, $C_{a}$ is the attractor of the i.f.s. $\{a x, a x+(1-a)\}$ in $\mathbb{R}$. When $\mathbb{X}=\mathbb{R}^{n}$ and the maps are similitudes, we say that $E$ is a self-similar set. See [4] for background on iterated function systems and self-similar sets.

Theorem 1 generalizes to arbitrary self-similar sets in $\mathbb{R}$ :

Theorem 2. Let $K$ (resp. $\left.K^{\prime}\right)$ be the attractor of the i.f.s. $\left\{r_{i} x+t_{i}\right\}_{i=1}^{n}$ (resp. $\left.\quad\left\{r_{i}^{\prime} x+t_{i}^{\prime}\right\}_{i=1}^{n^{\prime}}\right)$. If there exist $j, j^{\prime}$ such that $\log \left(\left|r_{j}\right|\right) / \log \left(\left|r_{j^{\prime}}^{\prime}\right|\right)$ is irrational, then

$$
\operatorname{dim}\left(K+K^{\prime}\right)=\min \left(\operatorname{dim}(K)+\operatorname{dim}\left(K^{\prime}\right), 1\right) .
$$

Next, we define two notions of resonance, and restate Theorem 2 using these notions. Let $\left\{r_{i} x+t_{i}\right\}_{i=1}^{n}$ and $\left\{r_{i}^{\prime} x+t_{i}^{\prime}\right\}_{i=1}^{n^{\prime}}$, be two i.f.s. consisting of similitudes, with attractors $K$ and $K^{\prime}$ respectively. We say that these two i.f.s. are algebraically resonant if for all $j \leq n$ and $j^{\prime} \leq n^{\prime}$, the ratio $\log \left|r_{j}\right| / \log \left|r_{j^{\prime}}^{\prime}\right|$ is rational. We say that the two i.f.s. are geometrically resonant if there is some $s>0$ such that

$$
\operatorname{dim}\left(K+s K^{\prime}\right)<\operatorname{dim}(K)+\operatorname{dim}\left(K^{\prime}\right) \leq 1 .
$$

Theorem 2 implies that two geometrically resonant iterated function systems must also be algebraically resonant. Below we will state a converse under a mild separation condition; see Theorem 4 .

The paper is structured as follows. In the remainder of this section we discuss some background related to Theorem 11. Section 2 contains a discrete version of the Marstrand Projection Theorem, which will be needed for the main proofs. The proof of Theorem 1 is in Section 3 , while Section 4 contains the proofs of the additional results stated at the end of this section. We finish the paper with some remarks and open questions in Section 5 .

1.1. Background. The study of the arithmetic sums of Cantor sets is a classical topic in real analysis, motivated in part by its rich applications to Diophantine approximation, the study of homoclinic bifurcations in dynamical systems, and other topics. The basic problem is to compute or estimate the size of the arithmetic sum $K+K^{\prime}$ in terms of the sizes of $K$ and $K^{\prime}$. 
"Size" can mean different things, but in this paper we focus on Hausdorff dimension.

Let $K, K^{\prime}$ be two compact subsets of the real line. Observe that

$$
K+K^{\prime} \text { is congruent to } \sqrt{2} P_{\pi / 4}\left(K \times K^{\prime}\right),
$$

where $P_{\theta}$ denotes the orthogonal projection onto the line $\{(t \cos \theta, t \sin \theta)\}_{t \in \mathbb{R}}$ making angle $\theta$ with the $x$-axis. Let $\gamma=\operatorname{dim}(K)+\operatorname{dim}\left(K^{\prime}\right)$. By Marstrand's projection theorem (see [12, Chapter 9) we have

$$
\begin{aligned}
& \gamma \leq 1 \Longrightarrow \operatorname{dim}\left(P_{\theta}\left(K \times K^{\prime}\right)\right)=\gamma \text { for almost every } \theta \in[0, \pi), \\
& \gamma>1 \Longrightarrow \mathcal{L}\left(P_{\theta}\left(K \times K^{\prime}\right)\right)>0 \text { for almost every } \theta \in[0, \pi),
\end{aligned}
$$

where $\mathcal{L}$ denotes Lebesgue measure. Unfortunately, Marstrand's theorem gives no information about specific values of $\theta$, and therefore cannot be directly applied to obtain information about sums of Cantor sets. However, it does support the heuristic principle that "typically",

$$
\operatorname{dim}\left(K+K^{\prime}\right)=\min \left(\operatorname{dim}(K)+\operatorname{dim}\left(K^{\prime}\right), 1\right) .
$$

Note that our definition of geometrical resonance for the iterated function systems that generate $K$ and $K^{\prime}$ (in the case where the sum of the dimensions is at most 1 ) requires at least one exception to the projection theorem for the set $K \times K^{\prime}$. We now discuss some earlier results that motivated our work.

The following result is due to Peres and Solomyak ([19], Theorem 1.1), generalizing an earlier result of Solomyak [23]:

Theorem 3. (Peres and Solomyak) Let

$$
\gamma(a)=\operatorname{dim}\left(C_{a}\right)=\log (2) / \log (1 / a) .
$$

Given a fixed compact set $K \subset \mathbb{R}$, the following holds for almost every $a \in(0,1 / 2)$ :

$$
\begin{aligned}
& \gamma(a)+\operatorname{dim}(K) \leq 1 \quad \Longrightarrow \operatorname{dim}\left(C_{a}+K\right)=\gamma(a)+\operatorname{dim}(K), \\
& \gamma(a)+\operatorname{dim}(K)>1 \Longrightarrow \mathcal{L}\left(C_{a}+K\right)>0 .
\end{aligned}
$$

In this theorem, the Cantor sets $C_{a}$ can be replaced by more general homogeneous self-similar sets (see [19] for details). If the ratio of $\log a$ and $\log b$ is rational, then

$$
\operatorname{dim}\left(C_{a}+C_{b}\right)<\operatorname{dim}\left(C_{a}\right)+\operatorname{dim}\left(C_{b}\right),
$$

This is folklore and can be seen as follows: if $\log a / \log b$ is rational, then there are $0<r<1$ and positive integers $m, n$ such that $a=r^{m}, b=r^{n}$. By iterating $n$ times the construction of $C_{a}$ and $m$ times the construction of $C_{b}$, we can decompose the product $C_{a} \times C_{b}$ into a union of cylinder sets whose convex hulls are squares. Moreover, two of these squares are $\left[0, r^{m n}\right] \times\left[1-r^{m n}, 1\right]$ and $\left[1-r^{m n}, 1\right] \times\left[0, r^{m n}\right]$. The projections of these squares on the line $\{(t, t)\}_{t \in \mathbb{R}}$ coincide. Thus, if we delete one of them from the construction of $C_{a} \times C_{b}$, and delete the corresponding square at 

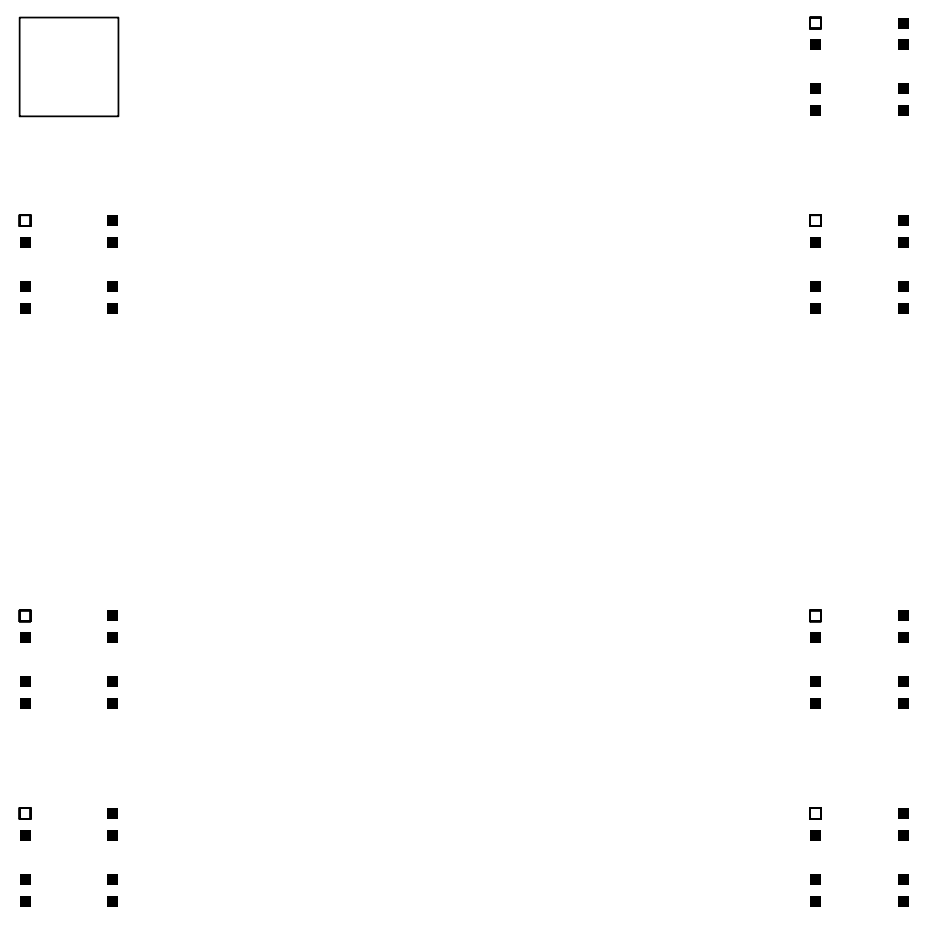

Figure 1. The structure of $C_{1 / 9} \times C_{1 / 3}$ : for each white square there is a black square of the same level, and with the same projection onto the line $\{(t, t)\}_{t \in \mathbb{R}}$. This results in a dimension drop for the projection, which is affinely equivalent to the arithmetic sum $C_{1 / 9}+C_{1 / 3}$.

every level, we obtain a self similar set $K \subset C_{a} \times C_{b}$ with $P_{\pi / 4}(K)=$ $P_{\pi / 4}\left(C_{a} \times C_{b}\right)$ and $\operatorname{dim}\left(P_{\pi / 4}(K)\right) \leq \operatorname{dim}(K)<\operatorname{dim}\left(C_{a} \times C_{b}\right)$. See Figure 1.1 for an illustration of this idea.

It follows that one cannot expect (2) to hold for all $a$, even when $K$ is also a central Cantor set. In [17] (as part of a more general framework) the dimension of the set of exceptions in (2) was estimated, but whether the set of exceptions (for $K=C_{b}$ ) is countable, was unknown until now. Eroğlu [3] proved that if $\operatorname{dim}\left(C_{a}\right)+\operatorname{dim}\left(C_{b}\right) \leq 1$, then the Hausdorff measure of the sum set $C_{a}+C_{b}$ in the $\operatorname{dimension} \operatorname{dim}\left(C_{a}\right)+\operatorname{dim}\left(C_{b}\right)$ is zero.

In a different direction, Moreira proved a result on the dimension of the sum of dynamically-defined, non-linear Cantor sets. This result was stated in [14, but the proof sketched there is incorrect; see [22] for a correct proof based on Moreira's ideas.

We remark that Moreira's Theorem has explicit checkable conditions, but it applies to nonlinear constructions; on the other hand the result of Peres and Solomyak applies to (affine) self-similar sets but it is an almosteverywhere type of result and gives no information about specific cases. 
Theorem 1 fills this gap by determining the exact set of exceptions to (2) in the case where $K$ is also a central Cantor set. In fact, our result holds for sums of general self-similar sets; see Theorem 2 below.

Our research was also inspired by some conjectures of H. Furstenberg [personal communication], who in the eighties asked about the validity of Theorem 1 in the particular case where $a^{-1}$ and $b^{-1}$ are integers which are not the power of a common integer. Other conjectures of Furstenberg, which are similar in spirit, remain open. For example, let $S$ be the one-dimensional Sierpinski gasket; i.e.

$$
S=\left\{\sum_{i=1}^{\infty} z_{i} 3^{-i}: z_{i} \in\{(0,0),(1,0),(0,1)\}\right\} .
$$

Furstenberg conjectured that $P_{\theta}(S)$ has dimension 1 but measure zero for all $\theta$ such that $\tan (\theta)$ is irrational. The part of the conjecture concerning measure was proved by Kenyon [10] and generalized by Lagarias and Wang in [11, but the dimension part is still open. Some related conjectures and results can be found in [6].

Palis [16] conjectured that, generically, if $K+K^{\prime}$ has positive Lebesgue measure, then it has nonempty interior. This conjecture motivated much of the research on this topic, and it was eventually answered positively in [15]. Although our work is not directly related to Palis' conjecture (indeed, we focus on the case where $\operatorname{dim}(K)+\operatorname{dim}\left(K^{\prime}\right)<1$, and in particular $K+K^{\prime}$ has zero measure), it can be seen as a continuation of the same line of research. We remark that Palis' conjecture restricted to self-similar sets is still open; i.e. it is not known whether sums of self-similar sets generically are either of zero measure or have nonempty interior.

The topological structure of $C_{a}+C_{b}$ when $\operatorname{dim}\left(C_{a}\right)+\operatorname{dim}\left(C_{b}\right)>1$ was investigated in [13] and [1]; the condition $\log b / \log a \notin \mathbb{Q}$ also arises naturally in this context.

1.2. Further results. The irrationality condition in Theorem 2 is equivalent to $\left\{\log \left|r_{i}\right|\right\} \cup\left\{\log \left|r_{i}^{\prime}\right|\right\}$ not being an arithmetic set (a subset of $\mathbb{R}$ is arithmetic if it is contained in some lattice $\alpha \mathbb{N}$ ). Our next result shows that, under the assumption that the Hausdorff dimension equals the so-called similarity dimension, Theorem 2 is sharp. We remark that this assumption is weaker than the well-known open set condition, see e.g. [4].

Theorem 4. Let $K$ (resp. $\left.K^{\prime}\right)$ be the attractor of the i.f.s. $\left\{r_{i} x+t_{i}\right\}_{i=1}^{n}$ (resp. $\left.\left\{r_{i}^{\prime} x+t_{i}^{\prime}\right\}_{i=1}^{n^{\prime}}\right)$, where $0<r_{i}, r_{i}^{\prime}<1$, and assume that the Hausdorff dimension of $K\left(\right.$ resp. $\left.K^{\prime}\right)$ is given by the only $\beta>0$ (resp. $\left.\beta^{\prime}>0\right)$ verifying $\sum_{i=1}^{n} r_{i}^{\beta}=$ 1 (resp. $\quad \sum_{i=1}^{n^{\prime}}\left(r_{i}^{\prime}\right)^{\beta^{\prime}}=1$ ). Suppose that the set $\left\{\log \left|r_{i}\right|\right\} \cup\left\{\log \left|r_{i}^{\prime}\right|\right\}$ is arithmetic. Then there exists some $s>0$ such that

$$
\operatorname{dim}\left(K+s K^{\prime}\right)<\operatorname{dim}(K)+\operatorname{dim}\left(K^{\prime}\right) .
$$


Using self-similarity, it is easy to show that if there is one $s$ such that (5) holds, then there are infinitely many. On the other hand, according to Marstrand's projection theorem, the set of parameters $s$ such that a dimension drop (5) occurs, has zero length. Furstenberg conjectured that this set is in fact countable, at least in the case where $K$ and $K^{\prime}$ are central Cantor sets, but our methods do not seem to yield progress on this problem.

A modification of the proof of Theorems 1 and 2 yields a new result on the projections of planar self-similar sets generated by an iterated function system that includes a scaled irrational rotation:

Theorem 5. Let $\left\{A_{i} x+d_{i}\right\}_{i=1}^{n}$ be a self-similar i.f.s. in $\mathbb{R}^{2}$ with attractor $E$. Suppose that each linear map $A_{i}$ is written as $\zeta_{i} R_{\theta_{i}} O_{i}$, where $\left|\zeta_{i}\right|<1$, the map $R_{\theta_{i}}$ is the rotation by angle $\theta_{i}$ and $O_{i}$ is either the identity or a reflection about the $x$-axis. Suppose that the group $G$ generated by $\left\{R_{\theta_{i}} O_{i}\right\}_{i=1}^{n}$ is such that the set $\left\{\theta: R_{\theta} \in G\right\}$ is dense in $[0, \pi)$. Then

$$
\operatorname{dim}\left(P_{\xi}(E)\right)=\min (\operatorname{dim}(E), 1) \quad \text { for all } \xi \in[0, \pi) .
$$

Figure 1.2 depicts one of the self-similar sets to which Theorem 5 applies. We remark that Eroğlu [2] proved that, under the assumptions of Theorem 5 , if the i.f.s. satisfies the open set condition and $\gamma=\operatorname{dim}(E) \leq 1$, then all projections $P_{\xi}(E)$ have zero $\gamma$-dimensional Hausdorff measure. Thus, in this case, the projections are smaller than the original set $E$ in measure, but not in dimension.

As part of the proofs of Theorems 2 and 5 , we will need the following proposition, which as far as we know is not in the literature.

Proposition 6. Let $\left\{A_{i} x+d_{i}\right\}_{i=1}^{n}$ be a self-similar i.f.s. on $\mathbb{R}^{p}$, where $p$ is either 1 or 2 , satisfying the open set condition, and let $E$ be its attractor.

Then for all $\varepsilon>0$, there exists an i.f.s. of the form $\left\{A x+z_{i}\right\}_{i=1}^{N}$ with attractor $H$, such that $H \subset E$, and

$$
\operatorname{dim}(H) \geq \operatorname{dim}(E)-\varepsilon
$$

\section{A discrete Marstrand Projection theorem}

We prove a discrete version of Marstrand's Theorem on projections. Similar results were obtained by Moreira (personal communication, see [22]) and Rams, in the context of intersection numbers, see [21] and references therein.

Proposition 7. Given constants $A>1, A_{1}, A_{2}>0$ and $0<\gamma<1$, there exists a constant $\delta$ such that the following holds:

Fix $\rho>0$. Let $\mathcal{Q}$ be a collection of disjoint closed convex subsets of the unit disk such that each element contains a disk of radius $A^{-1} \rho$ and is contained in a disk of radius $A \rho$. Suppose that $\mathcal{Q}$ has cardinality at least $\rho^{-\gamma} / A_{1}$, yet any disk of radius $\ell \in(\rho, 1)$ intersects at most $A_{2}(\ell / \rho)^{\gamma}$ elements of $\mathcal{Q}$. Then for any $\varepsilon>0$ there exists a set $J \subset[0, \pi]$ with the following properties:

(1) $\mathcal{L}([0, \pi] \backslash J) \leq \varepsilon$. 


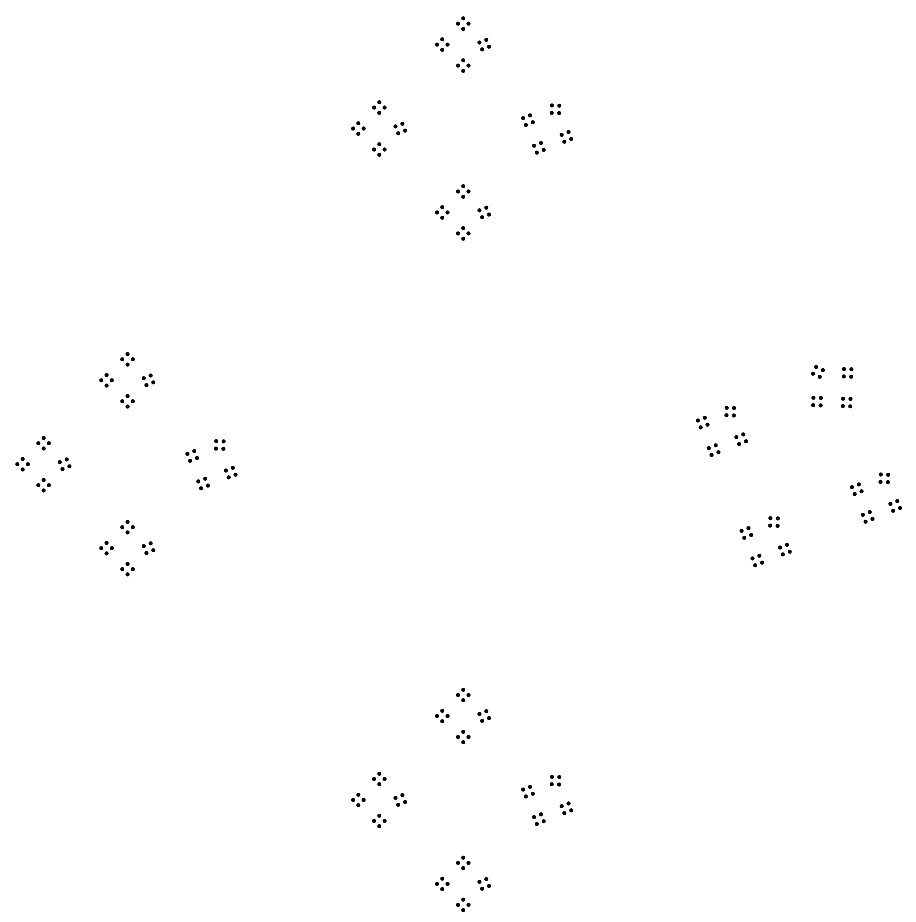

Figure 2. The attractor of an i.f.s. to which Theorem 5 applies. The dimension of this self-similar set is 1 . Theorem 5 says that all orthogonal projections are 1-dimensional as well.

(2) If $\theta \in J$, then there exists a subcollection $\mathcal{Q}_{1}$ of $\mathcal{Q}$ of cardinality at least $\varepsilon \delta|\mathcal{Q}|$ such that the orthogonal projections of the sets in $\mathcal{Q}_{1}$ onto a line with direction $\theta$ are all disjoint and $\rho$-separated.

(3) $J$ is a finite union of open intervals.

Proof. In the course of the proof, $c$ denotes a universal constant, and $A_{i}$ denote constants which depend only on $A, A_{1}, A_{2}$ and $\gamma$. Let $E$ be the union of all elements of $\mathcal{Q}$, and let $\mu$ be normalized Lebesgue measure on $E$. Consider the Riesz energy

$$
I_{1}(\mu)=\iint|z-w|^{-1} d \mu(z) d \mu(w) .
$$

We claim that

$$
I_{1}(\mu) \leq A_{4} \rho^{\gamma-1} .
$$

This is standard; we provide a proof below for completeness.

Assuming (6), we apply Theorem 9.9 in [12] with $n=2, m=1$, to obtain

$$
\int_{0}^{\pi}\left|P_{\theta} E\right|^{-1} d \theta \leq c I_{1}(\mu) \leq A_{5} \rho^{\gamma-1},
$$


where $P_{\theta} E$ are the projections of $E=\operatorname{Supp}(\mu)$ onto a line making angle $\theta$ with the $x$-axis, and $\left|P_{\theta} E\right|$ their length. Therefore, letting

$$
J_{*}=\left\{\theta:\left|P_{\theta} E\right|>\varepsilon A_{5}^{-1} \rho^{1-\gamma}\right\}
$$

we have $\mathcal{L}\left([0, \pi] \backslash J_{*}\right) \leq \varepsilon$.

Let $J=J(\delta)$ be the set of all $\theta \in[0, \pi)$ with the following property: there exists a subcollection $\mathcal{Q}_{1}=\mathcal{Q}_{1}(\theta)$ of $\mathcal{Q}$ of cardinality at least $\varepsilon \delta|\mathcal{Q}|$, such that the orthogonal projections of the sets in $\mathcal{Q}_{1}$ onto a line with direction $\theta$ are all disjoint and $\rho$-separated.

Due to the convexity of the elements of $\mathcal{Q}$, the set $J=J(\delta)$ is a finite union of open intervals. We claim that $J_{*} \subset J(\delta)$ for $\delta=(5 A+5)^{-1} A_{5}^{-1}$. To prove this, consider $\theta \in J_{*}$. By the definition of $J_{*}$, we can find in $P_{\theta} E$ at least $\delta \varepsilon \rho^{-\gamma}$ points $x_{j}$ that are $3(A+1) \rho$-separated. Choose for each of these points $x_{j}$ one element in $\mathcal{Q}$ with projection that contains $x_{j}$. This yields a family $\mathcal{Q}_{1} \subset \mathcal{Q}$, of cardinality at least $\delta \varepsilon \rho^{-\gamma}$, such that the projections $\left\{P_{\theta}(Q): Q \in \mathcal{Q}_{1}\right\}$ are at least $(A+1) \rho$-separated, as desired.

Proof of (6). We will need the following properties of $\mu$ :

(i) $A_{2}^{-1} A^{-4} \rho^{\gamma} \leq \mu(Q) \leq A_{1} A^{4} \rho^{\gamma}$ for any element $Q$ of $\mathcal{Q}$.

(ii) $\mu(D) \leq A_{7} \ell^{\gamma}$ for any disk $D$ of radius $\ell \in[\rho, 1]$.

(iii) $\mu \leq \bar{A}_{8} \rho^{\gamma-2} \mathcal{L}_{2}$, where $\mathcal{L}_{2}$ denotes two-dimensional Lebesgue measure.

To begin, observe that if $Q_{1}, Q_{2}$ are elements of $\mathcal{Q}$, then $\mu\left(Q_{1}\right) / \mu\left(Q_{2}\right) \leq A^{4}$. Since $\mathcal{Q}$ has between $\rho^{-\gamma} / A_{1}$ and $A_{2} \rho^{-\gamma}$ elements, (i) follows. If $D$ is a disk of radius $\ell$, where $\rho \leq \ell \leq 1$, then $D$ intersects at most $A_{2}(\ell / \rho)^{\gamma}$ elements of $\mathcal{Q}$, whence (ii) follows from (i) with $A_{7}=A_{1} A^{4} A_{2}$. Next, observe that

$$
\mathcal{L}_{2}(E) \geq\left(A_{1}^{-1} \rho^{-\gamma}\right)\left(A^{-2} \rho^{2}\right)=A_{1}^{-1} A^{-2} \rho^{2-\gamma},
$$

and from this we deduce that, for any Borel set $B \subset \mathbb{R}^{2}$,

$$
\mu(B)=\mu(B \cap E)=\mathcal{L}_{2}(E)^{-1} \mathcal{L}_{2}(B \cap E) \leq A_{1} A^{2} \rho^{\gamma-2} \mathcal{L}_{2}(B),
$$

which shows that (iii) holds with $A_{8}=A_{1} A^{2}$.

Now fix $w \in E$. Using (iii) we estimate

$$
\int_{|z-w| \leq \rho}|z-w|^{-1} d \mu(z) \leq A_{8} \rho^{\gamma-2} \int_{|u| \leq \rho}|u|^{-1} d u=2 \pi A_{8} \rho^{\gamma-1} .
$$

On the other hand, for $j \geq 1$ we can apply (ii) to obtain

$$
\mu\left(\left\{z:|z-w| \leq e^{j} \rho\right\}\right) \leq A_{7}\left(e^{j} \rho\right)^{\gamma},
$$

and from here we deduce

$$
\int_{e^{j-1} \rho<|z-w| \leq e^{j} \rho}|z-w|^{-1} d \mu(z) \leq A_{7}\left(e^{j} \rho\right)^{\gamma} e^{1-j} \rho^{-1}=e A_{7}\left(e^{j} \rho\right)^{\gamma-1} .
$$

Adding (7) and the sum of (8) over all $j \in \mathbb{N}$, we get

$$
\int|z-w|^{-1} d \mu(z) \leq A_{4} \rho^{\gamma-1}
$$


Finally, integrating over $w$ yields (6), and the proof is complete.

\section{Proof of Theorem 1}

We first discuss the main ideas of the proof of Theorem 1 full details follow below. Recall that Marstrand's theorem says that

$$
\operatorname{dim}\left(C_{a}+e^{t} C_{b}\right)=\min \left(\operatorname{dim}\left(C_{a}\right)+\operatorname{dim}\left(C_{b}\right), 1\right)
$$

for almost every $t \in \mathbb{R}$ (for reasons which will become apparent later, it will be useful to work in a logarithmic scale). Proposition 7 gives a discrete version of this: we decompose $C_{a} \times C_{b}$ into cylinder rectangles of size approximately $\rho \times \rho$. For $t \in \mathbb{R}$ consider the projection mapping $\Pi_{e^{t}}$. It will follow from Proposition 7 that there is a set $F_{0}$ of "good" values, whose complement has very small measure, such that for $t \in F_{0}$ a large number of rectangles have disjoint $\Pi_{e^{t}}$ projections.

Because of the homogeneity of $C_{a}, C_{b}$, all these cylinders are translates of each other, and they are pairwise disjoint. The idea now is to decompose these first cylinders into subcylinders of size approximately $\rho^{2} \times \rho^{2}$ and consider the set of "good" scales $F_{1}$ associated to them; because the cylinders are a (anisotropic) rescaling of $C_{a} \times C_{b}, F_{1}$ is of the form $F_{0}+\alpha_{1}$ for some $\alpha_{1} \in \mathbb{R}$. We continue this process inductively, to obtain sets of good scales $F_{n}$ after $n$ steps; we have $F_{n+1}=F_{n}+\alpha_{n}$ for some sequence $\left\{\alpha_{n}\right\}$. Notice that there is some latitude in choosing this sequence; the main idea is to exploit this freedom to pick the $\alpha_{n}$ in such a way that $t \rightarrow t+\alpha_{n}$ is essentially an irrational rotation on the circle. By construction the sets $F_{i}$ are robust: they can be taken to be a finite union of intervals. Then Weyl's equidistribution principle implies that for any specific angle $\theta$, there are many cylinders at many levels (more precisely, at a set of levels of well-defined density close to 1) whose images under $\Pi_{e^{t}}$ are disjoint. From here a standard application of the mass distribution principle gives the desired lower bound on the dimension.

We remark that the idea of using the discrete version of Marstrand's theorem and then iterating the process on each cylinder is due to Moreira. However, while he uses a recurrence result which requires a nonlinearity assumption, we go in a different direction by using Weyl's equidistribution principle. Furstenberg used Weyl's Theorem in a related setting in 6].

We now proceed to the details of the proof. We will establish a slightly more general version which will be needed to prove Theorem 2 .

Theorem 8. Let $K$ be the attractor of the i.f.s. $\left\{r x+t_{i}\right\}_{i=1}^{n}$, and let $K^{\prime}$ be the attractor of the i.f.s. $\left\{r^{\prime} x+t_{i}^{\prime}\right\}_{i=1}^{n^{\prime}}$.

Let $I, I^{\prime}$ be the convex hulls of $K, K^{\prime}$ respectively. Assume that the intervals $r I+t_{i}$ are pairwise disjoint, as are the intervals $r^{\prime} I^{\prime}+t_{i}^{\prime}$.

Assume further that $\log (|r|) / \log \left(\left|r^{\prime}\right|\right)$ is irrational.

Then

$$
\operatorname{dim}\left(K+K^{\prime}\right)=\min \left(\operatorname{dim}(K)+\operatorname{dim}\left(K^{\prime}\right), 1\right)
$$


The central Cantor sets $C_{a}$ satisfy the hypothesis of Theorem 8 , whence Theorem 1 is an immediate consequence of it.

Proof of Theorem 8.

Notation and remarks. The upper estimate

$$
\operatorname{dim}\left(K+K^{\prime}\right) \leq \min \left(\operatorname{dim}(K)+\operatorname{dim}\left(K^{\prime}\right), 1\right)
$$

is well known, so we only need to prove that the opposite inequality also holds.

If $\operatorname{dim}(K)+\operatorname{dim}\left(K^{\prime}\right) \geq 1$, then for any $\varepsilon>0$ we can, by iterating the original i.f.s. and throwing away some maps, find a subset $\widetilde{K}$ of $K$ such that the hypothesis of the Theorem still apply to the pair $\left(\widetilde{K}, K^{\prime}\right)$, and

$$
1-\varepsilon<\operatorname{dim}(\widetilde{K})+\operatorname{dim}\left(K^{\prime}\right)<1 .
$$

Thus we can assume without loss of generality that

$$
\gamma:=\operatorname{dim}(K)+\operatorname{dim}\left(K^{\prime}\right)<1 .
$$

Dilating both $K, K^{\prime}$ by the same factor and translating either of $K$ or $K^{\prime}$ does not affect either the hypothesis nor the result; therefore we can also assume that $I=[0,1]$ and $I^{\prime}=\left[0, e^{\tau}\right]$ for some $\tau \in \mathbb{R}$. Therefore it is enough to show that

$$
\operatorname{dim}\left(K+e^{\tau} K^{\prime}\right) \geq \operatorname{dim}(K)+\operatorname{dim}\left(K^{\prime}\right)-\eta
$$

for all $\eta>0$, under the additional assumption that the unit interval is the convex hull of both $K$ and $K^{\prime}$. We will fix $\tau$ for the rest of the proof.

Let $\Sigma^{*}$ be the family of all finite words $u=\left(u_{1}, \ldots, u_{k}\right)$ with $u_{i} \in$ $\{1, \ldots, n\}$; we define $\left(\Sigma^{\prime}\right)^{*}$ analogously. As customary, $|u|$ will denote the length of $u$. Concatenation of words will be denoted by juxtaposition; $1^{j}$ denotes the word consisting of $j$ consecutive ones.

Let $f_{i}(x)=r x+t_{i}, f_{i}^{\prime}(x)=r^{\prime} x+t_{i}^{\prime}$. For $u \in \Sigma^{*}, u^{\prime} \in\left(\Sigma^{\prime}\right)^{*}$ we will write

$$
f_{u}=f_{u_{1}} \cdots f_{u_{k}}, \quad f_{u^{\prime}}^{\prime}=f_{u_{1}^{\prime}}^{\prime} \cdots f_{u_{k^{\prime}}^{\prime}}
$$

where $|u|=k,\left|u^{\prime}\right|=k^{\prime}$. Also let $I(u)=f_{u}(I)$ and $I^{\prime}\left(u^{\prime}\right)=f_{u^{\prime}}^{\prime}(I)$. Finally, write $Q\left(u, u^{\prime}\right)=I(u) \times I^{\prime}\left(u^{\prime}\right)$. We underline that $f^{\prime}$ here is not a derivative.

Recall that $P_{\theta}$ is the orthogonal projection onto a line making angle $\theta$ with the $x$-axis. The projection mappings $(x, y) \rightarrow x+t y$ will be denoted by $\Pi_{t}$. A trivial but useful observation is that $P_{\theta}(\Lambda)$ and $\Pi_{\tan \theta}(\Lambda)$ are the same up to affine equivalence.

\section{Application of the discrete version of Marstrand's Theorem.}

In the course of the proof, $c$ will denote a constant which depends only on $K$ and $K^{\prime}$; its value may be different in each line.

Given $k \geq 0$, consider the families

$$
\begin{aligned}
& \mathcal{Q}_{k}=\left\{Q\left(u, u^{\prime}\right):|u|=k,\left|u^{\prime}\right|=k^{\prime}\right\}, \\
& \widetilde{\mathcal{Q}}_{k}=\left\{Q\left(u, u^{\prime}\right):|u|=k,\left|u^{\prime}\right|=k^{\prime}+1\right\},
\end{aligned}
$$


where $k^{\prime}$ is the largest integer such that $r^{k}<\left(r^{\prime}\right)^{k^{\prime}}$. Note that rectangles in $\mathcal{Q}_{k}$ have size $r^{k} \times M_{k} r^{k}$, where $1<M_{k}<1 / r^{\prime}$, and rectangles in $\widetilde{\mathcal{Q}}_{k}$ have size $r^{k} \times M_{k} r^{\prime} r^{k}$

Observe that $\left|\mathcal{Q}_{j}\right|>c r^{-j \gamma}$. This is well known; see for example [5, Proposition 7.4] and the remarks preceding it. Moreover, if $j<k$, then any $Q \in \mathcal{Q}_{j}$ contains at most $c r^{(j-k) \gamma}$ elements of $\mathcal{Q}_{k}$. Therefore if $S$ is any square of side $r^{j}$, then $S$ intersects a uniformly bounded number of elements of $\mathcal{Q}_{j}$, and combined with the previous remark we get that

$$
\left|\left\{Q \in \mathcal{Q}_{k}: Q \subset S\right\}\right| \leq c r^{(j-k) \gamma} .
$$

It follows from these observations that $\mathcal{Q}_{k}$ verifies the conditions of Proposition 7 for some $A, A_{1}, A_{2}$ which depend only on $K, K^{\prime}$, and in particular are independent of $k$.

Now pick some large integer $m$ and some small $\varepsilon>0$, and apply Proposition 7 to $\mathcal{Q}_{m}$ to obtain a set $J$ of "good" angles. Note that because every rectangle of $\widetilde{\mathcal{Q}}_{m}$ is contained in a rectangle of $\mathcal{Q}_{m}$, property (2) of $J$ in Proposition 7 is valid for $\widetilde{\mathcal{Q}}_{m}$ as well.

Write $\alpha=\log \left(M_{m}\right), \beta=\log \left(1 / r^{\prime}\right)$. A crucial fact is that $\alpha / \beta$ is irrational; this follows easily from the irrationality assumption. In particular, the map

$$
\mathbf{R}(x)=x+\alpha(\bmod \beta)
$$

is uniquely ergodic on $[0, \beta)$ endowed with normalized Lebesgue measure. We observe that $\mathbf{R}^{k}(0)=\log \left(M_{m k}\right)$; this fact will be useful later.

We will show that

$$
\operatorname{dim}\left(K+e^{\tau} K^{\prime}\right) \geq \gamma-\eta
$$

where $\eta$ depends on $m$ and $\varepsilon$, and can be made arbitrarily small by letting $m \rightarrow \infty, \varepsilon \rightarrow 0$.

If $\theta \in[0, \pi)$, then the orthogonal projection $P_{\theta}$ can be identified with the map $\Pi_{s}(x, y)=x+s y$, where $s=\tan (\theta)$. Since the map $\theta \rightarrow \log (\tan (\theta))$ is a diffeomorphism on any compact subset of the set of directions with positive and finite slope, it follows from Proposition 7 that there exists $\widetilde{F} \subset[\tau, \tau+\beta)$ and constants $L, \delta_{1}$ (independent of $m$ and $\varepsilon$ ) such that

(i) $\mathcal{L}([\tau, \tau+\beta) \backslash \widetilde{F}) \leq L \varepsilon$.

(ii) If $t \in \widetilde{F}$, then there exist $\mathcal{D}=\mathcal{D}(t) \subset \mathcal{Q}_{m}, \widetilde{\mathcal{D}}=\widetilde{\mathcal{D}}(t) \subset \widetilde{\mathcal{Q}}_{m}$ such that $|\mathcal{D}|>\delta_{1} \varepsilon r^{-m \gamma}$ and the family

$$
\left\{\Pi_{e^{t}}(Q): Q \in \mathcal{D}\right\}
$$

is $r^{m}$-separated; and analogous assertions hold for $\widetilde{D}$. '

(iii) $\widetilde{F}$ is a union of finitely many open intervals.

Finally, let $F=\widetilde{F}-\tau$.

Inductive construction. We will now use properties (i) and (ii) to inductively construct a tree $\mathcal{R}$ with vertices labeled by cylinder rectangles. 
The set of rectangles of level $j$ will be denoted by $\mathcal{R}_{j}$. We will prove that the tree has the following properties:

(A) If $Q$ is the parent of $Q^{\prime}$, then $Q^{\prime} \subset Q$.

(B) If $Q \in \mathcal{R}_{j}$, then $Q$ has size $r^{m j} \times \exp \left(\mathbf{R}^{j}(0)\right) r^{m j}$.

(C) The family $\left\{\Pi_{e^{\tau}}(Q): Q \in \mathcal{R}_{j}\right\}$ is disjoint and $r^{j m}$-separated.

(D) All rectangles of level $j$ have the same number of children $C_{j}$. Moreover,

$$
\begin{aligned}
& \mathbf{R}^{j}(0) \notin F \quad \Longrightarrow \quad C_{j}=1, \\
& \mathbf{R}^{j}(0) \in F \quad \Longrightarrow \quad C_{j} \geq \delta_{1} \varepsilon r^{-m \gamma} .
\end{aligned}
$$

(E) Each $Q \in \mathcal{R}_{j}$ is of the form $Q\left(v, v^{\prime}\right)$ for some $v \in \Sigma^{*}, v^{\prime} \in\left(\Sigma^{\prime}\right)^{*}$.

Let the root of the tree be the unit square. Define $\mathcal{R}_{1}$ as follows: if $\tau \in \widetilde{F}$, then $\mathcal{R}_{1}=\mathcal{D}(t)$; otherwise, $\mathcal{R}_{1}=\{\widehat{Q}\}$, where $\widehat{Q}$ is any element of $\mathcal{Q}_{m}$ (say, $\left.\widehat{Q}=Q\left(1^{m}, 1^{m^{\prime}}\right)\right)$. Property (B) follows from the definition of $\alpha$. Properties (A), (C), (D) and (E) are also clear.

Now assume that $\mathcal{R}_{j}$ has been defined verifying (A)-(E). We will consider two cases:

(I) $\mathbf{R}^{j}(0)+\alpha<\beta$.

(II) $\mathbf{R}^{j}(0)+\alpha>\beta$.

Let $\sigma=0$ if (I) holds, and $\sigma=1$ otherwise. Observe that

$$
\mathbf{R}^{j+1}(0)=\mathbf{R}^{j}(0)+\alpha-\sigma \beta .
$$

If $P, Q$ are rectangles define their product $Q \cdot P$ as $f_{Q} f_{P}\left(Q_{0}\right)$, where $Q_{0}$ is the unit square and $f_{P}, f_{Q}$ are the orientation preserving linear maps mapping $Q_{0}$ onto $P, Q$ respectively. Now given $Q \in \mathcal{R}_{j}$ we will define its set of offspring $C(Q)$ as follows: if $\mathbf{R}^{j}(0) \in F$, let

$$
C(Q)=\left\{\begin{array}{ll}
\left\{Q \cdot P: P \in \mathcal{D}\left(\mathbf{R}^{j}(0)+\tau\right)\right\} & \text { if case (I) holds } \\
\left\{Q \cdot P: P \in \widetilde{\mathcal{D}}\left(\mathbf{R}^{j}(0)+\tau\right)\right\} & \text { if case (II) holds }
\end{array} .\right.
$$

Otherwise, if $\mathbf{R}^{j}(0) \notin F$, then let $C(Q)=Q \cdot Q\left(1^{m}, 1^{m^{\prime}+\sigma}\right)$.

Property (A) is clear. Property (B) follows from (11) since each element of $\mathcal{R}_{j+1}$ has size

$r^{m j} r^{m} \times r^{m j} \exp \left(\mathbf{R}^{j}(0)\right) M_{m}\left(r^{\prime}\right)^{\sigma} r^{m}=r^{m(j+1)} \times \exp \left(\mathbf{R}^{j}(0)+\alpha-\sigma \beta\right) r^{m(j+1)}$.

If $\mathbf{R}^{j}(0) \notin F$, then property (C) is trivial, so we will assume that $\mathbf{R}^{j}(0) \in F$ or, in other words, $\mathbf{R}^{j}(0)+\tau \in \widetilde{F}$. It is enough to show that if $S_{1}, S_{2}$ are children of the same $Q \in \mathcal{R}_{j}$, then their projections are $r^{m(j+1)}$ separated. Moreover, by translating $Q$ if needed we can assume that $Q=r^{m j}(I \times$ $\left.\exp \left(\mathbf{R}^{j}(0)\right) I\right)$ where $I$ is the unit interval. Let $S_{i}=Q \cdot P_{i}$, where $P_{i}=J_{i} \times J_{i}^{\prime}$. 
We have:

$$
\begin{aligned}
\Pi_{e^{\tau}}\left(S_{i}\right) & =\Pi_{e^{\tau}}\left(Q \cdot P_{i}\right) \\
& =r^{j m} \Pi_{e^{\tau}}\left(\left(I \times \exp \left(\mathbf{R}^{j}(0)\right) I\right) \cdot\left(J_{i} \times J_{i}^{\prime}\right)\right) \\
& =r^{j m}\left(J_{i} \times \exp \left(\mathbf{R}^{j}(0)+\tau\right) J_{i}^{\prime}\right) \\
& =r^{j m} \Pi_{\exp \left(\mathbf{R}^{j}(0)+\tau\right)} P_{i} .
\end{aligned}
$$

By property (ii) of $\widetilde{F}$ the family $\left\{\Pi_{\exp \left(\mathbf{R}^{j}(0)+\tau\right)} P_{i}\right\}$ is $r^{m}$-separated; it follows that the family $\left\{\Pi_{e^{\tau}}\left(S_{i}\right)\right\}$ is $r^{(j+1) m}$-separated, as desired.

Property (D) follows immediately from Property (ii) above. Finally, Property $(\mathrm{E})$ is a direct consequence of the construction.

\section{Application of Weyl's equidistribution principle.}

We recall that $\widetilde{F}$, and hence $F$, is a finite union of open intervals. Therefore we can apply Weyl's equidistribution principle to get

$$
\lim _{j \rightarrow \infty} \frac{1}{j}\left|\left\{i<j: \mathbf{R}^{i}(0) \in F\right\}\right|=\frac{\mathcal{L}(F \cap[0, \beta))}{\mathcal{L}([0, \beta))} \geq 1-(L / \beta) \varepsilon .
$$

Let

$$
E_{\tau}=\bigcap_{j=1}^{\infty} \bigcup_{Q \in \mathcal{R}_{j}} \Pi_{e^{\tau}}(Q) .
$$

By property (A) this set is a countable intersection of nested nonempty compact sets, so $E_{\tau}$ is compact and nonempty. It follows from property (E) that $E_{\tau} \subset K+e^{\tau} K^{\prime}$.

We will estimate the dimension of $E_{\tau}$ in a standard way, by means of a Frostman measure. Let $\mu_{\tau}$ be the probability measure which assigns the same mass $\left|\mathcal{R}_{j}\right|^{-1}$ to all intervals $\Pi_{e^{\tau}}(Q)$ for $Q \in \mathcal{R}_{j}$. This measure is well defined thanks to properties (C) and (D), and is supported on $E_{\tau}$ by definition.

Let $x \in E_{\tau}$. Because of $(\mathrm{C})$ the interval $\left(x-r^{j m}, x+r^{j m}\right)$ intersects exactly one interval $\Pi_{e^{\tau}}(Q)$ with $Q \in \mathcal{R}_{j}$, so we have

$$
\mu_{\tau}\left(x-r^{j m}, x+r^{j m}\right) \leq \mu_{\tau}\left(\Pi_{e^{\tau}}(Q)\right)=\left|\mathcal{R}_{j}\right|^{-1} .
$$

On the other hand, using (D) once again we get that

$$
\log \left|\mathcal{R}_{j}\right| \geq\left|\left\{i<j: \mathbf{R}^{i}(0) \in F\right\}\right| \log \left(\delta_{1} \varepsilon r^{-m \gamma}\right) .
$$

Hence we deduce from (12) that if $j$ is large enough, then

$$
\log \mu_{\tau}\left(x-r^{j m}, x+r^{j m}\right) \leq j(1-(2 L / \beta) \varepsilon) \log \left(\delta_{1}^{-1} \varepsilon^{-1} r^{m \gamma}\right) .
$$

Thus from the mass distribution principle (see e.g. [5, Proposition 2.3]) we conclude that

$$
\operatorname{dim}\left(K+e^{\tau} K^{\prime}\right) \geq \operatorname{dim}\left(E_{\tau}\right) \geq \frac{(1-(2 L / \beta) \varepsilon) \log \left(\delta_{1}^{-1} \varepsilon^{-1} r^{m \gamma}\right)}{m \log r} .
$$


The right-hand side can be made arbitrarily close to $\gamma$ by letting $m \rightarrow \infty$, $\varepsilon \rightarrow 0$. Therefore the Hausdorff dimension of $K+e^{\tau} K^{\prime}$ must be at least $\gamma$, and this completes the proof.

\section{Remaining PROOFs}

Proof of Theorem 2 Let $K$ and $K^{\prime}$ be as in the statement of the theorem. We can assume without loss of generality that $\log \left(\left|r_{1}\right|\right) / \log \left(\left|r_{1}^{\prime}\right|\right) \notin \mathbb{Q}$.

Fix $\varepsilon>0$. Pick $\delta$ small enough so that for any covering $\left\{I_{j}\right\}$ of $K$ by intervals of diameter at most $3 \delta$, we have

$$
\sum_{j}\left|I_{j}\right|^{\operatorname{dim}(K)-\varepsilon}>1 .
$$

Let $I$ be the convex hull of $K$. Consider a covering of $K$ by cylinder intervals $f_{u}(I)$ of diameter between $r_{*} \delta$ and $\delta$, where $r_{*}=\min _{i}\left|r_{i}\right|$. Pick a maximal pairwise disjoint collection $\left\{f_{u_{j}}(I)\right\}_{j=1}^{N}$ among these cylinders. By maximality, if $I_{j}$ is the interval with the same center as $f_{u_{j}}(I)$ and length $\left|f_{u_{j}}(I)\right|+2 \delta$, then $\left\{I_{j}\right\}_{j=1}^{N}$ is a covering of $K$. Then we have

$$
\sum_{j=1}^{N}(3 \delta)^{\operatorname{dim}(K)-\varepsilon} \geq \sum_{j=1}^{N}\left|I_{j}\right|^{\operatorname{dim}(K)-\varepsilon}>1 .
$$

It follows that $N \geq c \delta^{\varepsilon-\operatorname{dim}(K)}$, where $c=3^{\varepsilon-\operatorname{dim}(K)}$. Let $\widetilde{K}$ be the attractor of the i.f.s. $\left\{f_{u_{j}}\right\}_{j=1}^{N}$. We have that $\widetilde{K} \subset K$ and

$$
\operatorname{dim}(\widetilde{K}) \geq \frac{\log N}{\left|\log \left(r_{*} \delta\right)\right|} \geq \frac{\log c+(\operatorname{dim}(K)-\varepsilon)|\log \delta|}{\left|\log \left(r_{*}\right)\right|+|\log \delta|} .
$$

Hence by taking $\delta$ very small we can ensure that $\operatorname{dim}(\widetilde{K})>\operatorname{dim}(K)-2 \varepsilon$. Moreover, all the intervals $f_{u_{j}}(I)$ are disjoint. In the same way we obtain an appropriate set $\widetilde{K}^{\prime} \subset K^{\prime}$. Therefore we can assume, without loss of generality, that the families

$$
\left\{r_{i} I+t_{i}\right\}_{i=1}^{n}, \quad\left\{r_{i}^{\prime} I^{\prime}+t_{i}^{\prime}\right\}_{i=1}^{n^{\prime}}
$$

are disjoint for some intervals $I, I^{\prime}$ (one can take these intervals to be the convex hulls of $K$ and $K^{\prime}$ ). At this point the irrationality condition may fail to hold.

We now apply Proposition 6 to $K$ and $K^{\prime}$, to obtain iterated function systems $\left\{g_{i}(x)\right\}_{i=1}^{N},\left\{g_{i}^{\prime}(x)\right\}_{i=1}^{N^{\prime}}$ with attractors $H, H^{\prime}$, where

$$
g_{i}(x)=\rho x+z_{i}, \quad g_{i}^{\prime}(x)=\rho^{\prime} x+z_{i}^{\prime},
$$

the dimensions of $H, H^{\prime}$ are very close to those of $K, K^{\prime}$, and $H \subset K, H^{\prime} \subset$ $K^{\prime}$. If $\log |\rho| / \log \left|\rho^{\prime}\right|$ is irrational we can apply Theorem 8 to $H+H^{\prime}$, and we are done. If not, then consider the new i.f.s.

$$
\left\{f_{1} \circ g_{i}(x)\right\}_{i=1}^{N}
$$


where $f_{1}(x)=r_{1} x+t_{1}$. The dimension of the attractor $\widetilde{H} \subset K$ is given by

$$
\frac{\log N}{-\left(\log |\rho|+\log \left|r_{1}\right|\right)}
$$

which, by picking $\rho$ small enough, can be made arbitrarily close to the dimension of $H$ (and hence to the dimension of $K$ ). Define $\widetilde{H}^{\prime}$ analogously. Since we are assuming that $\log (|\rho|) / \log \left(\left|\rho^{\prime}\right|\right) \in \mathbb{Q}$, and that $\log \left(\left|r_{1}\right|\right) / \log \left(\left|r_{1}^{\prime}\right|\right) \notin \mathbb{Q}$, it follows that $\widetilde{H}, \widetilde{H}^{\prime}$ verify the irrationality hypothesis and we can apply Theorem 8 to $\widetilde{H}+\widetilde{H}^{\prime}$. This completes the proof.

\section{Proof of Theorem 4.}

By assumption there exist $\xi>0$ and integers $\left\{a_{i}\right\},\left\{b_{i}\right\}$ such that $r_{i}=\xi^{a_{i}}$ and $r_{i}^{\prime}=\xi^{b_{i}}$.

We first consider the case in which all the $a_{i}$ are equal to some $a$ and all the $b_{i}$ are equal to some $b$. In this case $K+K^{\prime}$ is also self-similar and the proof is much easier; notice that algebraically resonant central Cantor sets fall into this case. By iterating the first i.f.s. $b$ times and the second i.f.s. $a$ times, and replacing $\xi$ by $\xi^{a b}$, we can assume that $a=b=1$. In this case the attractors are up to affine equivalence explicitly given by

$$
K=\left\{\sum_{i=1}^{\infty} d_{i} \xi^{i}: d_{i} \in\left\{t_{1}, \ldots, t_{n}\right\}\right\}, \quad K^{\prime}=\left\{\sum_{i=1}^{\infty} d_{i} \xi^{i}: d_{i} \in\left\{t_{1}^{\prime}, \ldots, t_{n^{\prime}}^{\prime}\right\}\right\} .
$$

Moreover, $\operatorname{dim}(K)=\log (n) / \log (1 / \xi)$, and $\operatorname{dim}\left(K^{\prime}\right)=\log \left(n^{\prime}\right) / \log (1 / \xi)$. Therefore, letting $D=\left\{t_{1}, \ldots, t_{n}\right\}$ and $D^{\prime}=\left\{t_{1}^{\prime}, \ldots, t_{n^{\prime}}^{\prime}\right\}$, we have

$$
K+s K^{\prime}=\left\{\sum_{i=1}^{\infty} d_{i} \xi^{i}: d_{i} \in D+s D^{\prime}\right\},
$$

whence

$$
\operatorname{dim}\left(K+s K^{\prime}\right) \leq \frac{\log \left|D+s D^{\prime}\right|}{\log (1 / \xi)}
$$

One can clearly take $s$ so that $\left|D+s D^{\prime}\right|<|D|\left|D^{\prime}\right|$; for example, let $s=$ $\left(t_{n}-t_{1}\right) /\left(t_{n^{\prime}}^{\prime}-t_{1}^{\prime}\right)$. For any such $s$, a dimension drop occurs.

We now consider the general case. We will use the same notation as in the proof of Theorem 8 . We claim that by iterating the constructions and reordering we can assume that $a_{1}=a_{2}=b_{1}=b_{2}$. Indeed, one can replace $f_{i}(i=1,2)$ by the collection of maps $\left\{f_{i} f_{u}:|u|=a_{3-i} b_{1} b_{2}-1\right\}$, and $f_{i}^{\prime}(i=1,2)$ by $\left\{f_{i}^{\prime} f_{u}^{\prime}:|u|=b_{3-i} a_{1} a_{2}-1\right\}$. This operation does not change the attractors $K$ and $K^{\prime}$. But now $f_{1} \circ f_{1}^{a_{2} b_{1} b_{2}-1}$ and $f_{2} \circ f_{2}^{a_{1} b_{1} b_{2}-1}$ are maps in the first i.f.s. (they are obtained by taking $i=1$ and $u$ a word consisting of all $1 \mathrm{~s}$, and then $i=2$ and $u$ a word consisting of all $2 \mathrm{~s}$, respectively), and they both have similarity ratio $\xi^{a_{1} a_{2} b_{1} b_{2}}$. Likewise, there are two maps in the second i.f.s. with this similarity ratio; this proves the claim. 
Denote the common value of $a_{1}, a_{2}, b_{1}, b_{2}$ by $\ell$. By a further translation and dilation we can also assume that the convex hull of both $K$ and $K^{\prime}$ is the unit interval.

After these simplifications, notice that $Q(1,1)$ and $Q(2,2)$ are squares of the same size, and we can choose $s$ so that $\Pi_{s}(Q(1,1))=\Pi_{s}(Q(2,2))$. By our assumption that all the maps are orientation-preserving and by selfsimilarity, it follows that

$$
\Pi_{s}\left(f_{1}(K) \times f_{1}^{\prime}\left(K^{\prime}\right)\right)=\Pi_{s}\left(f_{2}(K) \times f_{2}^{\prime}\left(K^{\prime}\right)\right) .
$$

More generally, if for some $u, u^{\prime}$ we have $r_{u}=r_{u^{\prime}}^{\prime}$, then

$$
\Pi_{s}\left(f_{(u 1)}(K) \times f_{\left(u^{\prime} 1\right)}^{\prime}\left(K^{\prime}\right)\right)=\Pi_{s}\left(f_{(u 2)}(K) \times f_{\left(u^{\prime} 2\right)}^{\prime}\left(K^{\prime}\right)\right) .
$$

Let $a$ be the g.c.d. of $\left\{a_{i}\right\}$, and $b$ the g.c.d. of $\left\{b_{i}\right\}$. Write also $A=$ $\max \left\{a_{i}\right\}, B=\max \left\{b_{i}\right\}$. By [9, Theorem 1.4.1], there is a smallest integer $M_{0}$ with the following property: all multiples of $a b$ that are greater than or equal to $M_{0}$ can be represented as a linear combination of $\left\{a_{i}\right\}$ with positive integral coefficients, and can also be represented as a linear combination of $\left\{b_{i}\right\}$ with positive integral coefficients. Pick some $M \geq M_{0}+\max (A, B)$ which is a multiple of $a b$.

Let $\Sigma=\{1, \ldots, n\}^{\mathbb{N}}$. Given $\omega \in \Sigma$ let

$$
\begin{aligned}
S_{j}(\omega) & =\sum_{i=1}^{j} a_{\omega_{i}} ; \\
n_{k}(\omega) & =\min \left\{j: S_{j}(\omega) \geq k M\right\} ; \\
L_{k}(\omega) & =S_{n_{k}}(\omega)-k M .
\end{aligned}
$$

All these definitions also apply to finite words of the appropriate length. Recall that the Hausdorff dimension of $K$ is given by the only $\beta>0$ such that

$$
\sum_{i=1}^{n} \xi^{\beta a_{i}}=1 .
$$

Endow $\Sigma$ with the Bernoulli measure $\mu$ for the probability vector $\left(\xi^{\beta a_{i}}\right)_{i=1}^{n}$. Define $\Sigma^{\prime}, S_{j}^{\prime}, n_{k}^{\prime}, L_{k}^{\prime}, \beta^{\prime}, \mu^{\prime}$ analogously.

Notice that $L_{k}$ can take the values $0, a, \ldots,(A / a-1) a$. Because of the way $M$ was defined, there is a finite sequence $\left\{j_{m}\right\}$ such that

$$
(k+1) M=S_{n_{k}}(\omega)+\sum a_{j_{m}} .
$$

Since $\mu$ is Bernoulli, this shows that there exist $\left\{p_{i}\right\}$, independent of $k$, such that

$$
\mu\left(S_{n_{k+1}}=(k+1) M \mid L_{k}=i a\right)=p_{i}, \quad \text { for } i=0,1, \ldots A / a-1,
$$

and analogously for $\mu^{\prime}$. Let $\nu=\mu \times \mu^{\prime}$; we will treat $\nu$ like a probability distribution according to which pairs of sequences are drawn at random. 
Consider the events

$$
\begin{aligned}
\Omega_{k} & =\left\{S_{n_{k}}=S_{n_{k}^{\prime}}^{\prime}=k M\right\} \\
\Xi_{k, i} & =\left\{\omega\left(n_{k}+1\right)=i, \omega^{\prime}\left(n_{k}^{\prime}+1\right)=i\right\} \quad(i=1,2) ; \\
\Theta_{k, i} & =\Omega_{k} \cap \Xi_{k, i} .
\end{aligned}
$$

Letting $p=\min \left(\left\{p_{i}\right\},\left\{p_{i}^{\prime}\right\}\right)$, it follows from (14) and independence that

$$
\nu\left(\Omega_{k}\right) \geq p^{2}, \quad \nu\left(\Xi_{k, i}\right)=\xi^{\ell\left(\beta+\beta^{\prime}\right)}, \quad \nu\left(\Theta_{k, i}\right) \geq \xi^{\ell\left(\beta+\beta^{\prime}\right)} p^{2} .
$$

Therefore

$$
\begin{aligned}
\nu\left(\Theta_{k+1,2} \cap \Theta_{k, 2}^{c}\right) & >\nu\left(\Theta_{k+1,2} \cap \Theta_{k, 1}\right) \\
& =\nu\left(\left(\Theta_{k+1,2} \mid S_{n_{k}+1}=S_{n_{k^{\prime}}^{\prime}+1}^{\prime}=k M+\ell\right) \cap \Theta_{k, 1}\right) \\
& \geq\left(\xi^{\ell\left(\beta+\beta^{\prime}\right)} p^{2}\right)^{2}=: q,
\end{aligned}
$$

where in the last inequality we used independence again. In particular, we have showed that $\nu\left(\Theta_{k+1,2}^{c} \mid \Theta_{k, 2}^{c}\right)<1-q$, and from this we deduce that

$$
\nu\left(\bigcap_{j=1}^{k} \Theta_{j, 2}^{c}\right)<(1-q)^{k} .
$$

We now come back to the geometric picture. Consider the following family:

$$
\mathcal{C}_{k}=\left\{u \in \Sigma^{*}: S_{|u|-1}(u)<k M \leq S_{|u|}(u)\right\} .
$$

Notice that $\left\{f_{u}(K): u \in \mathcal{C}_{k}\right\}$ is a covering of $K$; define $\mathcal{C}_{k}^{\prime}$ analogously. Let us call a pair $\left(u, u^{\prime}\right) \in \mathcal{C}_{k} \times \mathcal{C}_{k}^{\prime}$ redundant if $\left(u, u^{\prime}\right) \in \Theta_{j, 2}$ for some $j<k$; otherwise, let us call it essential.

We claim that

$$
\mathcal{E}_{k}=\left\{\Pi_{s}\left(Q\left(u, u^{\prime}\right)\right):\left(u, u^{\prime}\right) \in \mathcal{C}_{k} \times \mathcal{C}_{k}^{\prime} \text { is essential }\right\}
$$

is a covering of $\Pi_{s}\left(K \times K^{\prime}\right)$. Indeed, if $\left(u, u^{\prime}\right)$ is redundant, then it can be decomposed as $\left(v_{1} 2 v_{2}, v_{1}^{\prime} 2 v_{2}^{\prime}\right)$ where $r_{v_{1}}=r_{v_{1}^{\prime}}^{\prime}=\xi^{j M}$. Hence it follows from (13) that

$$
\Pi_{s}\left(K_{u} \times K_{u^{\prime}}^{\prime}\right) \subset \Pi_{s}\left(K_{\left(v_{1} 1\right)} \times K_{\left(v_{1}^{\prime} 1\right)}^{\prime}\right),
$$

and if we delete $\left(u, u^{\prime}\right)$ from $\mathcal{C}_{k} \times \mathcal{C}_{k}^{\prime}$ we still get a covering of the projection.

Notice that each interval in $\mathcal{E}_{k}$ has length bounded by $(s+1) \xi^{k M}$. Any essential pair $\left(u, u^{\prime}\right)$ has $\nu$-probability at least $\xi^{A \beta+B \beta^{\prime}} \xi^{k\left(\beta+\beta^{\prime}\right) M}$ of occurring. On the other hand, the probability of a pair in $\mathcal{C}_{k} \times \mathcal{C}_{k}^{\prime}$ being essential is, by (15), no more than $(1-q)^{k-1}$. It follows that there are at most

$$
\frac{1}{(1-q) \xi^{A \beta+B \beta^{\prime}}}\left(\left(\xi^{-\left(\beta+\beta^{\prime}\right) M}\right)(1-q)\right)^{k}
$$


essential pairs. As $k \rightarrow \infty$ we get coverings of $K+e^{s} K^{\prime}$ by intervals of arbitrarily small diameter. In the limit we can ignore the factors which do not depend on $k$, and we conclude that

$$
\operatorname{dim}\left(K+e^{s} K^{\prime}\right) \leq \frac{\left(\beta+\beta^{\prime}\right) M \log (1 / \xi)+\log (1-q)}{M \log (1 / \xi)}<\beta+\beta^{\prime},
$$

as desired.

Proof of Theorem 5. By proceeding like in the proof of Theorem 2, we can assume that $E$ is the attractor of an i.f.s. of the form $\left\{\zeta R_{\theta} x+d_{i}\right\}_{i=1}^{n}$, where $\theta / \pi$ is irrational, and the open set condition is satisfied. Write $f_{i}(x)=$ $\zeta R_{\theta} x+d_{i}$.

We can assume without loss of generality that $f_{i}(B) \subset B$ for all $i$, where $B$ is the unit ball. Fix some small $\varepsilon>0$ and some large $m$. We can apply Proposition 7 to the family $\mathcal{Q}=\left\{f_{u}(B):|u|=m\right\}$, with $\rho=\zeta^{m}$, $\gamma=\operatorname{dim}(E)$, and some $A, A_{1}, A_{2}$ which depend only on $E$. In this way we obtain a set of "good" angles $J$ verifying properties (1)-(3).

Let $\mathbf{R}$ be rotation by $m \theta$ on the circle. Because of the irrationality assumption, this is a uniquely ergodic transformation.

Fix some $\xi \in[0, \pi)$; we will use an inductive construction to show that

$$
\operatorname{dim}\left(P_{\xi}(E)\right) \geq \operatorname{dim}(E)-\eta,
$$

where $\eta$ depends on $m$ and $\varepsilon$ and can be made arbitrarily small. Our construction will yield a tree $\mathcal{R}$ with vertices labelled by disks, such that if $\mathcal{R}_{j}$ is the set of vertices at level $j$, then the following conditions hold:

(A) If $D$ is the parent of $D^{\prime}$, then $D^{\prime} \subset D$.

(B) If $D \in \mathcal{R}_{j}$, then $D=f_{u}(B)$ for some word $u$ of length $m j$. In particular, $D$ has radius $\zeta^{j m}$.

(C) The family $\left\{P_{\xi}(D): D \in \mathcal{R}_{j}\right\}$ is disjoint and $\zeta^{j m}$-separated.

(D) All vertices of level $j$ have the same number of children $C_{j}$. Moreover,

$$
\begin{aligned}
& \mathbf{R}^{j}(\xi) \notin J \quad \Longrightarrow \quad C_{j}=1, \\
& \mathbf{R}^{j}(\xi) \in J \quad \Longrightarrow \quad C_{j} \geq \varepsilon \delta \zeta^{-m \gamma} .
\end{aligned}
$$

Let $B$ be the root of the tree; i.e., the only vertex of level 0 . Now assume that a vertex $D$ of level $j$ has been defined; by property (E) we have $D=$ $f_{u}(B)$, where $u$ is a word of length $j m$. We consider two cases: if $\mathbf{R}^{j}(\xi) \notin J$, then $D$ has just one child, $f_{u 1^{m}}(B)$. If $\mathbf{R}^{j}(\xi) \in J$, then Proposition 7 guarantees that the family $\mathcal{Q}$ has a subcollection $\left\{f_{v_{i}}(B)\right\}_{i=1}^{M}$ such that all the projections $P_{\mathbf{R}^{j}(\xi)}\left(f_{v_{i}}(B)\right)$ are $\zeta^{m}$-separated, and $M \geq \varepsilon \delta \zeta^{-m \gamma}$. We define the set of offspring of $D$ to be $\left\{f_{u} \circ f_{v_{i}}(B)\right\}_{i=1}^{M}$.

Properties (A), (B), and (D) are immediate from the construction. To establish (C), it is enough to show that the projections of the offspring of a given $D \in \mathcal{R}_{j}$ are $\zeta^{(j+1) m}$-separated. Let $D=f_{u}(B)$; since $|u|=j m$ it follows that

$$
f_{u}(x)=\zeta^{j m} R_{\mathbf{R}^{j}(0)}(x)+d_{u}
$$


for some translation $d_{u}$. Using this we get

$$
\begin{aligned}
P_{\xi}\left(f_{u} \circ f_{v_{i}}(B)\right) & =P_{\xi}\left(\zeta^{j m} R_{\mathbf{R}^{j}(0)} f_{v_{i}}(B)+d_{u}\right) \\
& =\zeta^{j m} P_{\mathbf{R}^{j}(\xi)}\left(f_{v_{i}}(B)\right)+P_{\xi}\left(d_{u}\right) .
\end{aligned}
$$

This shows that $(\mathrm{C})$ holds as well.

Now let

$$
E_{\xi}=\bigcap_{j=1}^{\infty} \bigcup\left\{D: D \in \mathcal{R}_{j}\right\} .
$$

By properties (A) and (B) this set is well defined and contained in $P_{\xi}(E)$. Let $\mu_{\xi}$ be the probability measure which assigns equal mass to all intervals $P_{\xi}(D)$ for $D \in \mathcal{R}_{j}$; this is well defined because of properties (C) and (D); moreover, $\mu_{\xi}$ is supported on $E_{\xi}$. We estimate the dimension of $E_{\xi}$ using $\mu_{\xi}$ as a Frostman measure, applying Weyl's equidistribution principle to bound from above the $\mu_{\xi}$-measure of projections $P_{\xi}(D)$. This is done exactly as in the proof of Theorem 2, so we skip the details. In the end we obtain the estimate

$$
\operatorname{dim}\left(P_{\xi}(E)\right) \geq \frac{(1-\varepsilon)(\log (\varepsilon \delta)+m \gamma \log (1 / \zeta))}{m \log (1 / \zeta)}
$$

The right-hand side can be made arbitrarily close to $\gamma$, and this completes the proof.

Proof of Proposition 6. We will present the proof in the case $p=2$; the case $p=1$ is analogous but simpler. The linear maps $A_{i}$ can be decomposed as

$$
A_{i}(x)=r_{i} R_{\theta_{i}} O_{i}(x)
$$

where $\left|r_{i}\right|<1, R_{\theta_{i}}$ is rotation by angle $\theta_{i}$, and $O_{i}$ is either the identity or a reflection. Without loss of generality we can assume that $E$ does not contain reflections; i.o. all the $O_{i}$ are the identity. Indeed, if this is not the case, then, say, $O_{1}$ is a reflection. Then we can iterate the i.f.s. a large number of times, and then compose each of the resulting maps which contains a reflection with $A_{1}$. In this way we obtain a new self-similar set without reflections, which is contained in $E$ and with dimension arbitrarily close to the dimension of $E$.

Now let $\gamma$ be the only real number such that $\sum_{i} r_{i}^{\gamma}=1$. Since $E$ verifies the open set condition, this is also the Hausdorff dimension of $E$.

Let $\left\{e_{1}, \ldots, e_{n}\right\}$ be an orthonormal basis of $\mathbb{R}^{n}$. Consider the random walk which starts at 0 and moves from $x$ to $x+e_{i}$ with probability $r_{i}^{\gamma}$. Let $\mathbb{X}_{k}$ be the position of this random walk after $k$ steps. The mean of $\mathbb{X}_{k}$ is given by

$$
\mathbb{E} \mathbb{X}_{k}=\sum_{i=1}^{n} k r_{i}^{\gamma} e_{i}
$$


Let

$$
v=\sum_{i=1}^{n}\left(\left\lceil k r_{i}^{\gamma}\right\rceil\right) e_{i},
$$

and notice that we have $\left|v-\mathbb{E} \mathbb{X}_{k}\right| \leq \sqrt{n}$. It follows from [24, Chapter II, Proposition P9] that

$$
P\left(\mathbb{X}_{k}=v\right) \geq c(\sqrt{k})^{1-n},
$$

for some $c>0$ independent of $k$. Each of the paths which end in $v$ has probability

$$
\prod_{i=1}^{n} r_{i}^{\gamma v_{i}} \leq \prod_{i=1}^{n} r_{i}^{\gamma k r_{i}^{\gamma}}
$$

If $N_{k}$ is the number of such paths it follows from (16) that

$$
N_{k} \geq c(\sqrt{k})^{1-n} \prod_{i=1}^{n} r_{i}^{-k \gamma r_{i}^{\gamma}}
$$

Let

$$
\rho=\prod_{i=1}^{n} r_{i}^{v_{i}} \geq \prod_{i=1}^{n} r_{i} \prod_{i=1}^{n} r_{i}^{k r_{i}^{\gamma}}
$$

We can identify paths on the lattice with compositions of maps in the original i.f.s. Notice that all paths ending in $v$ are associated to a map of the form $A x+z_{i}$, where the similarity ratio of $A$ is $\rho$. The attractor $H$ of the i.f.s. generated by such maps is contained in $E$, and its dimension $\tau$ verifies

$$
N_{k} \rho^{\tau}=1 \Longrightarrow \tau=\frac{\log \left(N_{k}\right)}{\log (1 / \rho)} .
$$

From (17), (18) and (19) we obtain

$$
\tau \geq \frac{\log c+(1-n) \log \sqrt{k}+k \gamma \sum_{i=1}^{n} r_{i}^{\gamma} \log \left(1 / r_{i}\right)}{\sum_{i=1}^{n} \log \left(1 / r_{i}\right)+k \sum_{i=1}^{n} r_{i}^{\gamma} \log \left(1 / r_{i}\right)} .
$$

Since the right-hand terms dominate on both numerator and denominator, it follows that $\tau$ can be made arbitrarily close to $\gamma$ by taking $k$ large enough, completing the proof.

\section{CONCLUding REMARKS AND OPEN QUESTIONS}

(1) Sums of graph-directed attractors. Theorem 2 can be further generalized. The sets $K$ and $K^{\prime}$ can be attractors of more general graph-directed systems. In order to see this, one can either observe that the proof extends to this generality with minor variations, or just use the fact that attractors of graph-directed systems contain self-similar sets of arbitrarily close dimension. 
(2) Resonance as an equivalence relation. Let $\mathcal{I}$ denote the set of all self-similar i.f.s. satisfying the open set condition and such that the attractor has dimension at most 1/2. Algebraic resonance is clearly an equivalence relation on $\mathcal{I}$; it follows from our results that so is geometrical resonance. For more general compact sets there is no clear way to define resonance algebraically, but we can say that two compact sets $K, K^{\prime}$ of Hausdorff dimension at most $1 / 2$ are geometrically resonant if there is $s>0$ such that

$$
\operatorname{dim}\left(K+s K^{\prime}\right)<\operatorname{dim}(K)+\operatorname{dim}\left(K^{\prime}\right) .
$$

However, it turns out that this is not an equivalence relation. To see this, fix two very close numbers $a<b$ such that $C_{a}$ and $C_{b}$ have dimension less than $1 / 2$; moreover, from the proof of Theorem 4 it follows that we can ensure that the dimension drop in $C_{a}+C_{a}$ and $C_{b}+C_{b}$ is at least $4 \eta$, where $\eta=\operatorname{dim}\left(C_{b}\right)-\operatorname{dim}\left(C_{a}\right)$. Construct a compact set $K$ in the following way: pick a rapidly increasing sequence $n_{j}$. Follow the construction of $C_{a}$ for $n_{1}$ steps, then the construction of $C_{b}$ for $n_{2}$ steps, and so on. If this is done carefully, then one can ensure that $K$ has dimension $\operatorname{dim}\left(C_{a}\right)$ and

$$
\max \left(\operatorname{dim}\left(K+C_{a}\right), \operatorname{dim}\left(K+C_{b}\right)\right)<2 \operatorname{dim}\left(C_{b}\right)-2 \eta,
$$

whence $K$ is resonant to both $C_{a}$ and $C_{b}$, yet $C_{a}$ and $C_{b}$ need not be resonant.

(3) Uncountably many resonances. We can rephrase (2) in Theorem 3 as saying that a compact set cannot be resonant to a positive measure set of central Cantor sets. In light of the results of this paper, a natural question is whether an arbitrary compact set is resonant to at most countably many of the $C_{a}$. The answer, however, turns out to be negative. We sketch the construction of a compact set resonant to uncountably many central Cantor sets.

First of all, let us say that two compact sets $K, K^{\prime}$ are $\gamma$-resonant at scale $\rho$ if

$$
\mathcal{L}\left(K_{\rho}+K_{\rho}^{\prime}\right) \leq \rho^{-\gamma}
$$

where $K_{\rho}, K_{\rho}^{\prime}$ denote the $\rho$-neighborhoods of $K, K^{\prime}$. Pick $a_{1}>a_{2}$ very close to each other so that, for some $\eta>0$,

(i) $\operatorname{dim}\left(C_{a_{i}}\right)<1 / 2$.

(ii) $\operatorname{dim}\left(C_{a_{i}}\right) \in B_{\varepsilon / 10}(\eta)$.

$$
2 \operatorname{dim}\left(C_{a_{i}}\right)-\operatorname{dim}\left(C_{a_{i}}+C_{a_{i}}\right)>10 \varepsilon, \quad i=1,2 .
$$

We construct the desired compact set $K$ as follows (this is different from the set constructed in the previous remark): starting from the unit interval, follow the construction of $C_{a_{1}}$ for $n_{1}$ steps, so that at scale $\rho_{1}=a_{1}^{n_{1}}$ the sets $K$ and $C_{a_{1}}$ are $(2 \eta-2 \varepsilon)$-resonant. Observe that there exists a small interval $I_{1}$ around $a_{1}$ such that $K$ and $C_{a}$ 
are $(2 \eta-\varepsilon)$-resonant at scale $\rho_{1}$ for all $a \in I_{1}$; we can assume that $a_{2} \notin I_{1}$. Pick any $a_{11}<a_{12}<a_{1}$ in $I_{1}$.

Next we follow the construction of $C_{a_{2}}$ for $n_{2}$ steps, with $n_{2}>>$ $n_{1}$. In this way we can find a $\rho_{2}<<\rho_{1}$ such that $C_{a_{2}}$ and $K$ are $(2 \eta-\varepsilon)$-resonant at scale $\rho_{2}$. We find an interval $I_{2}$ around $a_{2}$, disjoint from $I_{1}$, and points $a_{21}>a_{22}>a_{2}$ as above.

We repeat this process for $a_{i j}$ to find intervals $I_{i j}$ and points $a_{i j k} \in$ $I_{i j}$ such that at some very small scales, $\rho_{i j}$ the sets $K$ and $C_{a_{i j}}$ are $(2 \eta-\varepsilon)$-resonant. We continue this construction inductively. Then if we let

$$
\mathcal{A}=\bigcap_{k=1}^{\infty} \bigcup_{i_{1}, \ldots, i_{k} \in\{1,2\}} I_{i_{1} \ldots i_{k}},
$$

it follows from the construction that if $a \in \mathcal{A}$, then $K$ and $C_{a}$ are $(2 \eta-\varepsilon)$-resonant at arbitrarily small scales. This implies that $C_{a}+K$ has dimension at most $2 \eta-\varepsilon$. Also, $\operatorname{dim}(K) \geq \eta-\varepsilon / 10$. We conclude that

$$
\begin{aligned}
\operatorname{dim}\left(C_{a}+K\right) & <(\eta-\varepsilon / 10)+(\eta-\varepsilon / 10)-4 \varepsilon / 5 \\
& <\operatorname{dim}\left(C_{a}\right)+\operatorname{dim}(K)-\varepsilon / 2 .
\end{aligned}
$$

The uncountable set of resonances in this example is of dimension zero. We do not know if there exists a compact set $K$ which is geometrically resonant to the central Cantor set $C_{a}$ for a set of parameters $a$ of positive dimension.

(4) The case $\operatorname{dim}\left(C_{a}\right)+\operatorname{dim}\left(C_{b}\right)>1$. In this paper we focused on the case where the sum of the dimensions is at most 1 . We do prove that if $\operatorname{dim}\left(C_{a}\right)+\operatorname{dim}\left(C_{b}\right)>1$ and $\log b / \log a$ is irrational, then $\operatorname{dim}\left(C_{a}+C_{b}\right)=1$. However, in light of Marstrand's projection theorem and Theorem 3(ii), it is natural to conjecture that $C_{a}+C_{b}$ actually has positive measure.

By analogy with the general results on intersections with lines (See [12, Chapter 10]) and Theorem 1.2 in [19], we also conjecture that, under the irrationality assumption, for all $\theta \in(0, \pi) \backslash\{\pi / 2\}$ there is a set of positive measure of lines with direction $\theta$ which intersect $C_{a} \times C_{b}$ in a set of $\operatorname{dimension} \operatorname{dim}\left(C_{a}\right)+\operatorname{dim}\left(C_{b}\right)-1$. Note that this would imply that $C_{a}+C_{b}$ has positive measure.

Acknowledgement. We are grateful to Hillel Furstenberg and Boris Solomyak for introducing us to these problems and for many enlightening discussions. We also thank Kemal Ilgar Eroğlu and the referee for helpful comments.

\section{REFERENCES}

[1] C. A. Cabrelli, K. E. Hare, and U. M. Molter. Sums of Cantor sets yielding an interval. J. Aust. Math. Soc., 73(3):405-418, 2002. 
[2] K. I. Eroğlu. On planar self-similar sets with a dense set of rotations. Annales Academiae Scientiarum Fennicae Mathematica, to appear. available at arXiv:math.CA/0603181, 2007.

[3] K. I. Eroğlu. On the arithmetic sums of cantor sets. Nonlinearity, 20:1145-1161, 2007.

[4] K. Falconer. Fractal geometry. John Wiley \& Sons Ltd., Chichester, 1990. Mathematical foundations and applications.

[5] K. Falconer. Techniques in fractal geometry. John Wiley \& Sons Ltd., Chichester, 1997.

[6] H. Furstenberg. Intersections of Cantor sets and transversality of semigroups. In Problems in analysis (Sympos. Salomon Bochner, Princeton Univ., Princeton, N.J., 1969), pages 41-59. Princeton Univ. Press, Princeton, N.J., 1970.

[7] M. Keane, K. Simon, and B. Solomyak. The dimension of graph directed attractors with overlaps on the line, with an application to a problem in fractal image recognition. Fund. Math., 180(3):279-292, 2003.

[8] M. Keane, M. Smorodinsky, and B. Solomyak. On the morphology of $\gamma$-expansions with deleted digits. Trans. Amer. Math. Soc., 347(3):955-966, 1995.

[9] J. G. Kemeny and J. L. Snell. Finite Markov chains. The University Series in Undergraduate Mathematics. D. Van Nostrand Co., Inc., Princeton, N.J.-Toronto-LondonNew York, 1960.

[10] R. Kenyon. Projecting the one-dimensional Sierpinski gasket. Israel J. Math., 97:221238, 1997.

[11] J. C. Lagarias and Y. Wang. Tiling the line with translates of one tile. Invent. Math., 124(1-3):341-365, 1996.

[12] P. Mattila. Geometry of sets and measures in Euclidean spaces, volume 44 of Cambridge Studies in Advanced Mathematics. Cambridge University Press, Cambridge, 1995. Fractals and rectifiability.

[13] P. Mendes and F. Oliveira. On the topological structure of the arithmetic sum of two Cantor sets. Nonlinearity, 7(2):329-343, 1994.

[14] C. G. T. d. A. Moreira. Sums of regular Cantor sets, dynamics and applications to number theory. Period. Math. Hungar., 37(1-3):55-63, 1998. International Conference on Dimension and Dynamics (Miskolc, 1998).

[15] C. G. T. d. A. Moreira and J.-C. Yoccoz. Stable intersections of regular Cantor sets with large Hausdorff dimensions. Ann. of Math. (2), 154(1):45-96, 2001.

[16] J. Palis. Homoclinic orbits, hyperbolic dynamics and dimension of Cantor sets. In The Lefschetz centennial conference, Part III (Mexico City, 1984), volume 58 of Contemp. Math., pages 203-216. Amer. Math. Soc., Providence, RI, 1987.

[17] Y. Peres and W. Schlag. Smoothness of projections, Bernoulli convolutions, and the dimension of exceptions. Duke Math. J., 102(2):193-251, 2000.

[18] Y. Peres, W. Schlag, and B. Solomyak. Sixty years of Bernoulli convolutions. In Fractal geometry and stochastics, II (Greifswald/Koserow, 1998), volume 46 of Progr. Probab., pages 39-65. Birkhäuser, Basel, 2000.

[19] Y. Peres and B. Solomyak. Self-similar measures and intersections of Cantor sets. Trans. Amer. Math. Soc., 350(10):4065-4087, 1998.

[20] M. Pollicott and K. Simon. The Hausdorff dimension of $\lambda$-expansions with deleted digits. Trans. Amer. Math. Soc., 347(3):967-983, 1995.

[21] M. Rams. Generic behavior of iterated function systems with overlaps. Pacific J. Math., 218(1):173-186, 2005.

[22] P. Shmerkin. Moreira's theorem on the sum of regular Cantor sets. Preprint, 2006.

[23] B. Solomyak. On the measure of arithmetic sums of Cantor sets. Indag. Math. (N.S.), 8(1):133-141, 1997.

[24] F. Spitzer. Principles of random walk. Springer-Verlag, New York, second edition, 1976. Graduate Texts in Mathematics, Vol. 34. 
Yuval Peres, Microsoft Research, Redmond and Departments of Statistics and Mathematics, University of California, Berkeley.

E-mail address: peres@stat.Berkeley.edu

Pablo Shmerkin, Departments of Mathematics and Statistics, University of JYVÄSKYLÄ.

E-mail address: shmerkin@maths.jyu.fi 